\title{
ORIGINAL
}

\section{Early extubation followed by immediate noninvasive ventilation vs. standard extubation in hypoxemic patients: a randomized clinical trial}

Rosanna Vaschetto ${ }^{1,2}$, Federico Longhini ${ }^{3}$, Paolo Persona ${ }^{4}$, Carlo Ori $^{5}$, Giulia Stefani ${ }^{5}$, Songqiao Liu ${ }^{6}$, Yang $\mathrm{Yi}^{6}$, Weihua $\mathrm{Lu}^{7}$, Tao Yu${ }^{7}$, Xiaoming Luo ${ }^{8}$, Rui Tang ${ }^{8}$, Maoqin Li ${ }^{9}$, Jiaqiong Li ${ }^{9}$, Gianmaria Cammarota ${ }^{1}$, Andrea Bruni ${ }^{10}$, Eugenio Garofalo ${ }^{10}$, Zhaochen Jin ${ }^{11}$, Jun Yan ${ }^{11}$, Ruiqiang Zheng ${ }^{12}$, Jingjing Yin ${ }^{12}$, Stefania Guido ${ }^{1}$, Francesco Della Corte ${ }^{1,2}$, Tiziano Fontana ${ }^{13}$, Cesare Gregoretti ${ }^{14}$, Andrea Cortegiani ${ }^{14}$, Antonino Giarratano ${ }^{14}$, Claudia Montagnini ${ }^{1}$, Silvio Cavuto ${ }^{15}$, Haibo Qiu ${ }^{6}$ and Paolo Navalesi ${ }^{10^{*}}$

(c) 2018 Springer-Verlag GmbH Germany, part of Springer Nature and ESICM

\begin{abstract}
Purpose: Noninvasive ventilation (NIV) may facilitate withdrawal of invasive mechanical ventilation (i-MV) and shorten intensive care unit (ICU) length of stay (LOS) in hypercapnic patients, while data are lacking on hypoxemic patients. We aim to determine whether NIV after early extubation reduces the duration of i-MV and ICU LOS in patients recovering from hypoxemic acute respiratory failure.
\end{abstract}

Methods: Highly selected non-hypercapnic hypoxemic patients were randomly assigned to receive NIV after early or standard extubation. Co-primary end points were duration of i-MV and ICU LOS. Secondary end points were treatment failure, severe events (hemorrhagic, septic, cardiac, renal or neurologic episodes, pneumothorax or pulmonary embolism), ventilator-associated pneumonia (VAP) or tracheobronchitis (VAT), tracheotomy, percent of patients receiving sedation after study enrollment, hospital LOS, and ICU and hospital mortality.

Results: We enrolled 130 consecutive patients, 65 treatments and 65 controls. Duration of i-MV was shorter in the treatment group than for controls [4.0 (3.0-7.0) vs. 5.5 (4.0-9.0) days, respectively, $p=0.004]$, while ICU LOS was not significantly different [8.0 (6.0-12.0) vs. $9.0(6.5-12.5)$ days, respectively ( $p=0.259)]$. Incidence of VAT or VAP ( $9 \%$ vs. $25 \%, p=0.019)$, rate of patients requiring infusion of sedatives after enrollment (57\% vs. 85\%, $p=0.001)$, and hospital LOS, 20 (13-32) vs. 27(18-39) days $(p=0.043$ ) were all significantly reduced in the treatment group compared with controls. There were no significant differences in ICU and hospital mortality or in the number of treatment failures, severe events, and tracheostomies.

Conclusions: In highly selected hypoxemic patients, early extubation followed by immediate NIV application reduced the days spent on invasive ventilation without affecting ICU LOS.

Keywords: Noninvasive ventilation, Hypoxemia, Extubation, Acute respiratory failure, Weaning

\footnotetext{
*Correspondence: pnavalesi@unicz.it

${ }^{10}$ Anestesia e Rianimazione, Dipartimento di Scienze Mediche e Chirurgiche, Università "Magna Graecia", Viale Europa (Loc. Germaneto), Catanzaro, Italy

Full author information is available at the end of the article
}

Rosanna Vaschetto and Federico Longhini contributed equally. 


\section{Introduction}

Patients with severe acute respiratory failure (ARF) often require intubation and invasive mechanical ventilation (i-MV). Although a life-saving intervention, $\mathrm{i}-\mathrm{MV}$ is prone to complications and side effects [1].

Noninvasive ventilation (NIV) can be used at an early stage of ARF to prevent the need for $\mathrm{i}-\mathrm{MV}$ and its associated risks. NIV may improve arterial blood gases (ABGs) and reduces the work of breathing in patients with both hypercapnic [2] and hypoxemic [3] ARF.

There is evidence supporting NIV for treatment of hypercapnic ARF secondary to chronic obstructive pulmonary disease (COPD) [4], cardiogenic pulmonary edema [5], and ARF following abdominal surgery [6].

Data available on the use of NIV as a means to facilitate the process of liberation from i-MV in non-hypercapnic hypoxemic ARF patients are presently limited to a small pilot trial [8], which makes any recommendation in favor of this approach or exclusion of potential harm impossible at this time [4]. No multicenter randomized controlled trial (RCT) on the use of NIV to facilitate the process of liberation from invasive mechanical ventilation only in non-hypercapnic hypoxemic patients is presently available.

We therefore designed this multicenter randomized controlled trial to assess whether early extubation followed by immediate NIV application would reduce the duration of i-MV and ICU length of stay (LOS) in a population of patients recovering from an episode of hypoxemic ARF, but still dependent on inspiratory pressure support and high levels of positive end-expiratory pressure (PEEP) as opposed to a conventional approach with the endotracheal tube in place.

\section{Methods}

\section{Trial design and oversight}

The present trial is an investigator-initiated, parallelgroup, multicenter RCT. A blocked randomization, with a block size of 10, was generated using a computergenerated allocation sequence held by an investigator not involved in the study enrollment, who indicated the group of assignment for each center in sealed, opaque numbered envelopes. The study was conducted from 13 October 2013 to 19 October 2016 in nine ICUs of academic hospitals, i.e., six in the Chinese Republic and three in Italy. The trial was conducted in accordance with the Declaration of Helsinki. The study was approved by all local Ethics Committees. Written informed consent was obtained for all patients. The trial was prospectively registered on 4 October 2013 at the Australian New Zealand Clinical Trial Registry (ACTRN12613001114730).

\section{Take-home message}

Few data are available on the use of noninvasive ventilation to facilitate extubation in hypoxemic non-hypercapnic acute respiratory failure patients. With this multicenter randomized trial, we show that early extubation followed by noninvasive ventilation application reduces the duration of invasive ventilation without significantly affecting intensive care unit length of stay and is associated with decreased occurrence of ventilator-associated pneumonia and tracheobronchitis, a reduced rate of patients requiring sedation after enrollment, and shorter hospital length of stay, without significantly affecting mortality. Surgical patients, as opposed to medical patients, seem to benefit most.

We followed the CONSORT recommendations concerning the report of randomized trials [9]. An external data monitor committee supervised data collection.

\section{Patients}

We considered every intubated patient meeting all the following inclusion criteria eligible: (1) age $\geq 18$ years; (2) invasive mechanical ventilation for at least $48 \mathrm{~h}$; (3) pressure support ventilation (PSV) with a total applied pressure, i.e., PEEP + inspiratory support $\leq 25 \mathrm{cmH}_{2} \mathrm{O}$ with PEEP level between 8 and $13 \mathrm{cmH}_{2} \mathrm{O}$; (4) the ratio between the partial pressure of oxygen and fraction of inspired oxygen $\left(\mathrm{PaO}_{2} / \mathrm{FiO}_{2}\right)$ between 200 and $300 \mathrm{mmHg}$ with $\mathrm{FiO}_{2} \leq 0.6$; (5) $\mathrm{PaCO}_{2} \leq 50 \mathrm{mmHg}$ and $\mathrm{pH} \geq 7.35$; (6) respiratory rate $(\mathrm{RR}) \leq 30 / \mathrm{min}$; (7) tidal volume $(\mathrm{VT})<8 \mathrm{ml} / \mathrm{kg}$ of ideal body weight (IBW); (8) core temperature $<38.5{ }^{\circ} \mathrm{C}$; (9) Glasgow Coma Scale $(\mathrm{GCS})=10 \mathrm{~T}$, i.e., the highest possible value in intubated patients; (10) cough on suctioning and need for tracheo-bronchial suctioning $<2$ per hour. Patients were excluded if they met one or more of the following criteria: (1) hemodynamic instability, i.e., systolic arterial pressure $<90 \mathrm{mmHg}$ despite adequate filling; (2) use of vasoactive agents, i.e., vasopressin, epinephrine at any dosage, dopamine, or dobutamine $>5 \mu \mathrm{g} / \mathrm{kg} / \mathrm{min}$, and norepinephrine $>0.2 \mu \mathrm{g} /$ $\mathrm{kg} / \mathrm{min}$; (3) life-threatening arrhythmias or electrocardiographic signs of ischemia; (4) presence of sepsis [10]; (5) ARF secondary to neurologic disorders, status asthmaticus, COPD, and cardiogenic pulmonary edema; (6) presence of tracheotomy; (7) uncontrolled vomiting; (8) two or more new organ failures; (9) body mass index $\geq 30 \mathrm{~kg} /$ $\mathrm{m}^{2} ;(10)$ documented history or suspicion of obstructive sleep apnea; (11) enrollment in other research protocols; (12) denied consent.

\section{Protocol}

All ICU patients underwent daily screening for study recruitment during the morning round. After enrollment, patients were allocated to either early extubation and NIV application (treatment) or standard extubation 
(control). All centers used ICU ventilators equipped with dedicated NIV software (Servo-I, Maquet Critical Care, Solna Sweden; EVITA 4, Draeger, Lubeck, Germany). A detailed description of the sedation protocol is provided in the online supplement.

Patients randomized to the intervention group were extubated without performing spontaneous breathing trials (SBT), and NIV was immediately applied with the same PEEP and inspiratory support set during i-MV, either oral-nasally or with a full-face mask or helmet, as detailed in the online supplement. When a mask was used, the occurrence of an expiratory VT between 6 and $8 \mathrm{ml} / \mathrm{kg}$ of IBW was verified. To achieve this target, adjustments of the inspiratory support were allowed. PEEP and PS were decreased by $2 \mathrm{cmH}_{2} \mathrm{O}$ every $2 \mathrm{~h}$ to a minimum of 8 and $10 \mathrm{cmH}_{2} \mathrm{O}$, respectively [8], as schematically reported in Fig. 1. A detailed description of the protocol for reduction of PEEP and PS is presented in the online supplement. When $\mathrm{PaO}_{2} /$ $\mathrm{FiO}_{2}$ exceeded $250 \mathrm{mmHg}$ with PEEP $8 \mathrm{cmH}_{2} \mathrm{O}$ and PS $10 \mathrm{cmH}_{2} \mathrm{O}$, a 30-min unsupported SBT was attempted with oxygen supplementation through a Venturi mask at $\mathrm{FiO}_{2}$ of 0.35 . NIV was interrupted if at the end of the unsupported SBT all the following occurred: $\mathrm{pH} \geq 7.35$, $\mathrm{PaCO}_{2} \leq 50 \mathrm{mmHg}$ and $\mathrm{PaO}_{2} \geq 70 \mathrm{mmHg}, \mathrm{RR} \leq 30$ breaths/min, absence of dyspnea, respiratory accessory muscle recruitment, and paradoxical abdominal motion.

In the control group, PEEP and PS were titrated as already described for the treatment group. At the minimum level of 8 and $10 \mathrm{cmH}_{2} \mathrm{O}$ of PEEP and PS, respectively, a partially supported SBT was conducted if $\mathrm{PaO}_{2} /$ $\mathrm{FiO}_{2}$ was $>250 \mathrm{mmHg}$. The SBT consisted of $30 \mathrm{~min}$ of breathing through the endotracheal tube with both PEEP and PS set at $5 \mathrm{cmH}_{2} \mathrm{O}$. Patients were extubated soon after SBT completion if $\mathrm{RR} \leq 30$ breaths/min, $\mathrm{pH} \geq 7.35$, $\mathrm{PaCO}_{2} \leq 50 \mathrm{mmHg}$, and $\mathrm{PaO}_{2} \geq 70 \mathrm{mmHg}$ with an $\mathrm{FiO}_{2}$ of 0.35 , without dyspnea, respiratory accessory muscle recruitment, and paradoxical abdominal motion. Once extubated, prophylactic NIV was used for for 6-12 h for all patients considered at risk for post-extubation respiratory failure [11], as detailed in the online supplement. Oxygen therapy was administered through a Venturi mask after NIV withdrawal in the treatment group or extubation in the control group.

In the control group, treatment failure was defined as need for noninvasive continuous positive airway pressure (nCPAP), NIV, or reintubation within the $48 \mathrm{~h}$ following extubation. Prophylactic NIV to prevent post-extubation respiratory failure was not considered treatment failure. In the treatment group, treatment failure was defined as need for nCPAP, NIV, or reintubation within $48 \mathrm{~h}$ after NIV withdrawal.
In both groups, patients who failed because of dyspnea with $7.30>\mathrm{pH}<7.35$ and $45 \mathrm{mmHg}<\mathrm{PaCO}_{2}<50 \mathrm{mmHg}$ underwent a "rescue" attempt of NIV before intubation. Similarly, in the case of hypoxemia only (i.e., $\mathrm{PaO}_{2} /$ $\mathrm{FiO}_{2}<200 \mathrm{mmHg}$ ), nCPAP was attempted. Predefined criteria for re-intubation are provided in the online supplement.

\section{Study end points}

The co-primary outcomes for comparing the two groups were the time spent on i-MV and ICU LOS. Secondary outcomes were (1) treatment failure; (2) occurrence of at least one of the following severe events: intervening hemorrhagic, septic, cardiac, renal [13], or neurologic (ischemic or hemorrhagic stroke) episodes, pneumothorax, or pulmonary embolism; (3) rate of ventilatorassociated pneumonia (VAP) or ventilator-associated tracheobronchitis (VAT); (4) tracheotomy; (5) rate of patients requiring sedation after study inclusion; (6) hospital LOS; (7) ICU and hospital mortality. VAT and VAP are more extensively defined in the online supplement.

\section{Statistical analysis}

Sample size calculation was obtained with nQuery Advisor software, version 7.0. Since the study had two primary objectives of equal relevance, defined a priori, the sample size required to achieve each of them was calculated separately and the highest number considered. Based on data from our previous work [8], we estimated a sample of 65 patients per group necessary for determination, with a power of $80 \%$ and a $5 \%$ two-sided level of significance. The null hypothesis was that in the treatment group mean ICU LOS was equal to that in the control group, i.e., 21 days, while the alternative hypothesis was that ICU LOS means were 15 and 21 days for the treatment and control group, respectively. Standard deviations were 11 and 13 days, respectively. We decided to compare the two groups with a $t$ test with Satterthwaite correction. After analyzing for the primary end point variables skewness, kurtosis, and normality distribution using the Shapiro-Wilk test and equality of variances by the Levene test, we decided on the Mann-Whitney test. An intention-to-treat analysis was used. To exclude effects of the center and country, we used a mixed effect linear regression model providing an estimate of the association between primary outcomes and treatment groups with the random intercept being center and country, as detailed in the online supplement.

Kaplan-Meier curves, depicting the two groups for the time from intubation to liberation (1) from i-MV and (2) from any form of mechanical ventilation, i.e., invasive 


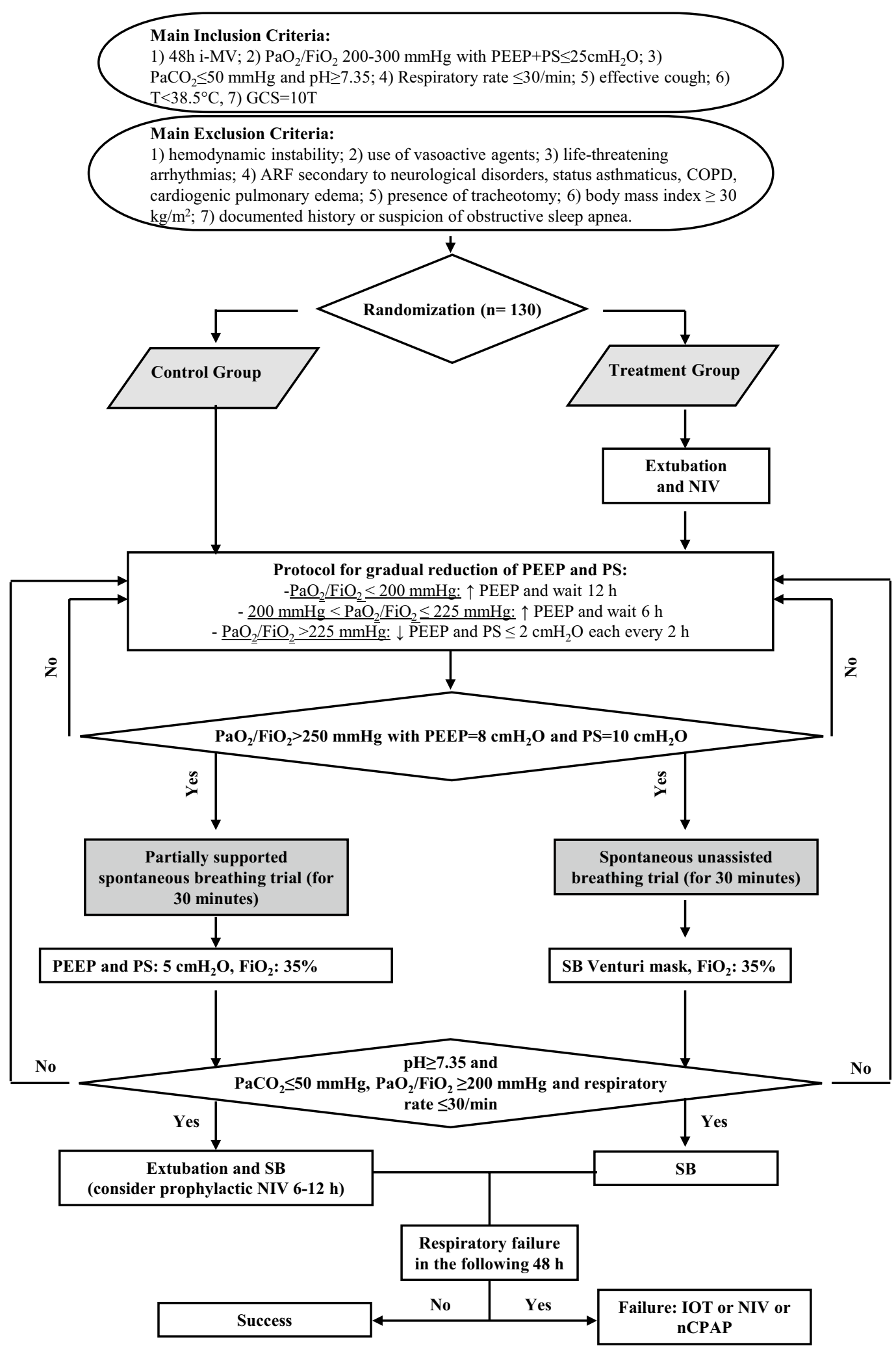

Fig. 1 Protocol flow chart. $\mathrm{FiO}_{2}$ inspired oxygen fraction. $\mathrm{PaO}_{2} / \mathrm{FiO}_{2}$ ratio of arterial oxygen partial pressure to fractional inspired oxygen; $P E E P$ positive end-expiratory pressure; PS pressure support; SB spontaneous breathing; IOT oro-tracheal intubation; NIV noninvasive ventilation; $n C P A P$ noninvasive continuous positive airway pressure; COPD chronic obstructive pulmonary disease; ARF acute respiratory failure 
and noninvasive, were determined and compared using the log-rank test.

Data analysis was performed using STATA software, version 14. Data are expressed as median \pm interquartile range. Frequency distributions are compared by two tailed chi-square test or Fisher's exact test, as indicated. $p$ values $<0.05$ were considered significant.

\section{Results}

We enrolled 130 patients, 65 randomized to undergo early extubation and immediate NIV and 65 receiving standard extubation. Figure 2 shows the flow of patients through the trial and detailed in the online supplement.

Data on primary outcomes were available for all 130 patients. We encountered some violations of the protocol, as detailed in the online supplement, which were analyzed according to the intention to treat (E-Results). Groups were similar with respect to anthropometric characteristics, severity scores at ICU admission, and comorbidities (eTable 1). The main reason for instituting $\mathrm{i}-\mathrm{MV}$ and the number of days of $\mathrm{i}-\mathrm{MV}$ pre-enrollment were similar between groups (Table 1). The initial settings of the ventilators were as follows: median PEEP 8 $\mathrm{cmH}_{2} \mathrm{O}\left(8-10 \mathrm{cmH}_{2} \mathrm{O}\right)$ for both groups; median pressure support $10 \mathrm{cmH}_{2} \mathrm{O}\left(10-12 \mathrm{cmH}_{2} \mathrm{O}\right)$ for both groups. Median $\mathrm{PaO}_{2} / \mathrm{FiO}_{2}$ and $\mathrm{PaCO}_{2}$ obtained with those settings were $248 \mathrm{mmHg}(221-278 \mathrm{mmHg})$ and $232 \mathrm{mmHg}$ $(218-269 \mathrm{mmHg})$ and $40 \mathrm{mmHg}(36-44 \mathrm{mmHg})$ and $39 \mathrm{mmHg}(35-44 \mathrm{mmHg})$, respectively. Normal $\mathrm{pH}$ values were present in both groups. The duration of mechanical ventilation before inclusion was similar, i.e., 4 (3-7) and 4 (3-6) days, for controls and treatments, respectively. Five patients in both groups attempted NIV before intubation, while no patient had been re-intubated before protocol inclusion.

Doses of vasoactive drugs on the day of extubation are presented in the online supplement. Arterial blood gas parameters at $\mathrm{T} 0, \mathrm{~T} 1, \mathrm{~T} 12$, and $\mathrm{T} 24$ are also presented in the online supplement. $\mathrm{PaO}_{2} / \mathrm{FiO}_{2}, \mathrm{PaCO}_{2}$, and $\mathrm{pH}$ were not different at each time point between the two groups (eTable 2).

\section{Primary outcomes}

Compared with standard extubation, NIV after early extubation significantly reduced the days spent on $\mathrm{i}-\mathrm{MV}$, i.e., $5.5(4.0-9.0)$ vs. $4.0(3.0-7.0)$ days, $p=0.004$, while ICU length of stay was not different between the two groups: $9.0(6.5-12.5)$ vs. $8.0(6.0-12.0)$ days, $p=0.259$ (Table 2). Compared with the intention-to-treat analysis, the results of the per-protocol analysis resulted in similar results (online supplement).
A post hoc analysis was performed, according to the diagnosis at ICU entrance, i.e., medical or surgical (including post-surgical and polytrauma patients). Medical patients did not show significant differences between the two groups with respect to both duration of i-MV and ICU LOS. Conversely, surgical patients in the treatment group displayed a shorter length of i-MV compared with controls, 3.0 (2.0-6.0) days vs. 5.4 (3.8-8.9) days, $p=0.004$, and shorter ICU stay, $6.0(5.0-8.3)$ vs. 8.5 (6.313.5) days, $p=0.036$.

\section{Secondary outcomes}

Treatment failures, occurrence of severe events, and number of tracheostomies were not different between groups (Table 2). We had five failures (3 reintubations, 1 $\mathrm{NIV}$, and $1 \mathrm{nCPAP}$ ) in the treatment group and 12 failures (7 reintubations, 3 NIVs, and 2 nCPAPs) in controls $(p=0.069)$. Only one of these patients, enrolled in the control group, died. Severe events occurred in 13 (20\%) and $18(28 \%)$ of patients in the treatment and control group, respectively $(p=0.303)$. Four controls received tracheostomy $(p=0.119)$. VAP and VAT occurred in two and four patients, respectively, in the treatment group, while in nine and seven patients, respectively, in the control group $(p=0.019)$. Sedatives and analgesic drugs were still administered after randomization in $57 \%$ and $85 \%$ of patients in treated patients and controls, respectively $(p=0.001)$. Hospital LOS was significantly decreased $(p=0.043)$ in the treatment group [20 (13-32) days] compared with controls [27(18-39) days]. ICU and hospital mortality was not different between groups (Table 2).

\section{Length of ventilation according to Kaplan-Meier analysis}

The Kaplan-Meier curve indicating the time from intubation to liberation from invasive ventilation is depicted in Fig. 3a. The median time of liberation from invasive ventilation was 4 days ( $95 \%$ CI $3-5$ days) in the treatment group and 5 days ( $95 \%$ CI 4-6 days) in controls, log-rank $p=0.019$. Considering the time to liberation from any form of mechanical ventilation, i.e., invasive and noninvasive, (Fig. $3 \mathrm{~b}$ ), the median time was 5.5 days $(95 \% \mathrm{CI}$ 4.1-7.0 days) in the treatment group and 6.0 days (95\% CI 5.0-7.0 days) in controls, log-rank $p=0.172$. The median time spent on NIV in the treatment group was 1 $(0.5-1.5)$ day.

\section{Discussion}

In this multicenter RCT study, conducted in patients recovering from an episode of non-hypercapnic hypoxemic ARF, NIV after early extubation reduced the days spent on i-MV without significantly affecting ICU length of stay. Treatment was associated with a 


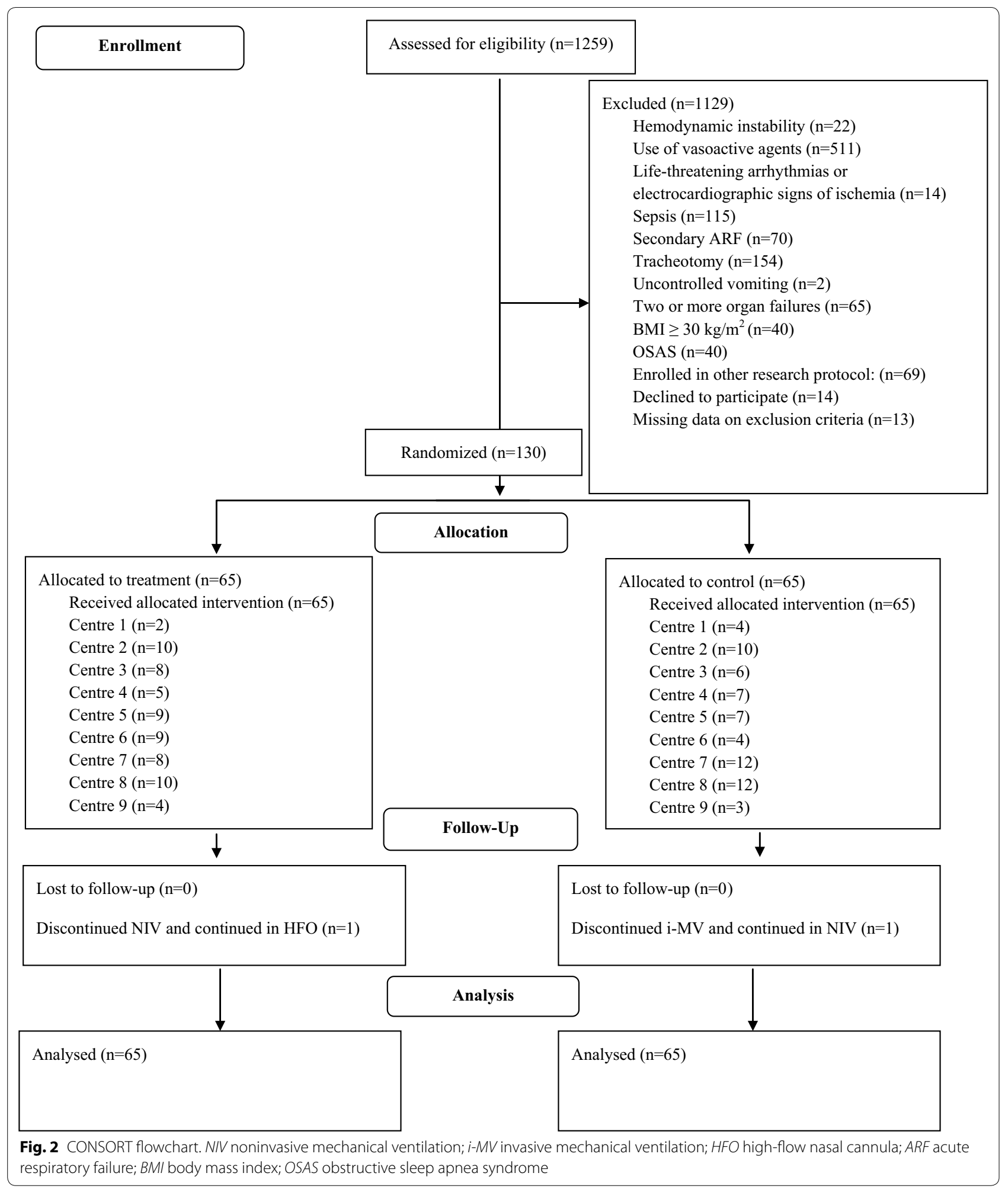

decreased rate of VAP or VAT, reduced need for sedation, and shorter hospital LOS, while mortality was not different between groups. Post-hoc analysis suggests that surgical patients are more likely to benefit from this clinical approach than those with medical diseases. These results are of potential major clinical interest.

Several studies proved NIV efficacy in reducing the duration of $\mathrm{i}-\mathrm{MV}$ in intubated patients affected by 


\section{Table 1 Clinical characteristics of the patients at enrollment}

\begin{tabular}{|c|c|c|}
\hline & Control $(n=65)$ & Treatment $(n=65)$ \\
\hline \multicolumn{3}{|l|}{ Main causes of i-MV, $n(\%)$} \\
\hline ARDS & $3(4.6 \%)$ & $6(9.2 \%)$ \\
\hline Pneumonia & $12(18.5 \%)$ & $15(23 \%)$ \\
\hline Septic shock & $3(4.6 \%)$ & $4(6.1 \%)$ \\
\hline Polytrauma & $12(18.5 \%)$ & $14(21.5 \%)$ \\
\hline Postoperative abdominal surgery & $12(18.5 \%)$ & $9(13.8 \%)$ \\
\hline Postoperative vascular surgery & $2(3.1 \%)$ & $2(3.1 \%)$ \\
\hline Postoperative thoracic surgery & $2(3.1 \%)$ & / \\
\hline Gastrointestinal bleeding & $3(4.6 \%)$ & $5(7.7 \%)$ \\
\hline Cerebral bleeding & $4(6.1 \%)$ & $1(1 \%)$ \\
\hline Pancreatitis & $2(3.1 \%)$ & l \\
\hline Others & $10(16.4 \%)$ & $9(13.8 \%)$ \\
\hline Days of i-MV pre-protocol, median (interquartile range) & $4(3-7)$ & $4(3-6)$ \\
\hline Days of NIV pre-protocol, median (interquartile range), $n=5$ in each group & $1(1-1)$ & $1(0.5-1)$ \\
\hline PEEP $\left(\mathrm{cmH}_{2} \mathrm{O}\right)$, median (interquartile range) & $8(8-10)$ & $8(8-10)$ \\
\hline Pressure support $\left(\mathrm{cmH}_{2} \mathrm{O}\right)$, median (interquartile range) & $10(10-12)$ & $10(10-12)$ \\
\hline Respiratory rate (breath/min), median (interquartile range) & $18(16-21)$ & $18(16-21)$ \\
\hline $\mathrm{FiO}_{2}(\%)$, median (interquartile range) & $40(35-45)$ & $40(40-40)$ \\
\hline $\mathrm{PaO}_{2} / \mathrm{FiO}_{2}(\mathrm{mmHg})$, median (interquartile range) & $248(221-278)$ & $232(218-269)$ \\
\hline pH, median (interquartile range) & $7.45(7.42-7.48)$ & $7.45(7.41-7.49)$ \\
\hline $\mathrm{PaCO}_{2}(\mathrm{mmHg})$, median (interquartile range) & $40(36-44)$ & $39(35-44)$ \\
\hline
\end{tabular}

i-MV invasive mechanical ventilation; NIV noninvasive mechanical ventilation; $A R D S$ acute respiratory distress syndrome; $P E E P$ positive end-expiratory pressure; FiO ${ }_{2}$ inspired fraction of oxygen; $\mathrm{PaO}_{2} / \mathrm{FiO}_{2}$ oxygen arterial partial pressure to inspired fraction ratio; $\mathrm{PaCO}_{2}$ arterial partial pressure of carbon dioxide

\section{Table 2 Primary and secondary outcomes}

\begin{tabular}{|c|c|c|c|}
\hline Primary outcomes & Control & Treatment & $p$ value \\
\hline \multicolumn{4}{|l|}{ Days of i-MV, median (interquartile range) } \\
\hline Overall & $5.5(4.0-9.0)(n=65)$ & $4.0(3.0-7.0)(n=65)$ & $0.004^{\mathrm{a}}$ \\
\hline Medical & $6.0(4.0-9.3)(n=29)$ & $5.0(3.0-8.0)(n=39)$ & $0.107^{a}$ \\
\hline Surgical & $5.4(3.8-8.9)(n=36)$ & $3.0(2.0-6.0)(n=26)$ & $0.004^{\mathrm{a}}$ \\
\hline \multicolumn{4}{|l|}{ ICU stay, median (interquartile range) } \\
\hline Overall & $9.0(6.5-12.5)(n=65)$ & $8.0(6.0-12.0)(n=65)$ & $0.259^{a}$ \\
\hline Medical & $9(6.5-13.0)(n=29)$ & $9.0(6.0-14.0)(n=39)$ & $0.970^{\mathrm{a}}$ \\
\hline Surgical & $8.5(6.3-13.5)(n=36)$ & $6.0(5.0-8.3)(n=26)$ & $0.036^{\mathrm{a}}$ \\
\hline Secondary outcomes & Control $(n=65)$ & Treatment $(n=65)$ & $p$ value \\
\hline Treatment failure, $n(\%)$ & $12(18 \%)$ & $5(8 \%)$ & $0.069^{b}$ \\
\hline Severe events, $n(\%)$ & $18(28 \%)$ & $13(20 \%)$ & $0.303^{b}$ \\
\hline Tracheostomy, n (\%) & $4(6 \%)$ & 0 & $0.119^{c}$ \\
\hline VAT and VAP, $n(\%)$ & $16(25 \%)$ & $6(9 \%)$ & $0.019^{b}$ \\
\hline Use of sedatives, $n(\%)$ & $55(85 \%)$ & $37(57 \%)$ & $0.001^{b}$ \\
\hline Hospital LOS, median (interquartile range) & $27(18-39)$ & $20(13-32)$ & $0.043^{\mathrm{a}}$ \\
\hline ICU mortality, $n(\%)$ & $1(1.5 \%)$ & $3(4.6 \%)$ & $0.619^{c}$ \\
\hline Hospital mortality, $n(\%)$ & $4(6.2 \%)$ & $6(9.2 \%)$ & $0.510^{b}$ \\
\hline
\end{tabular}

i-MV invasive mechanical ventilation; ICU intensive care unit; VAP ventilator-associated pneumonia; VAT ventilator-associated tracheobronchitis; LOS length of stay

a $p$ value obtained with Mann-Whitney test

b $p$ value obtained with chi-square test

c $p$ value obtained with Fisher exact test 

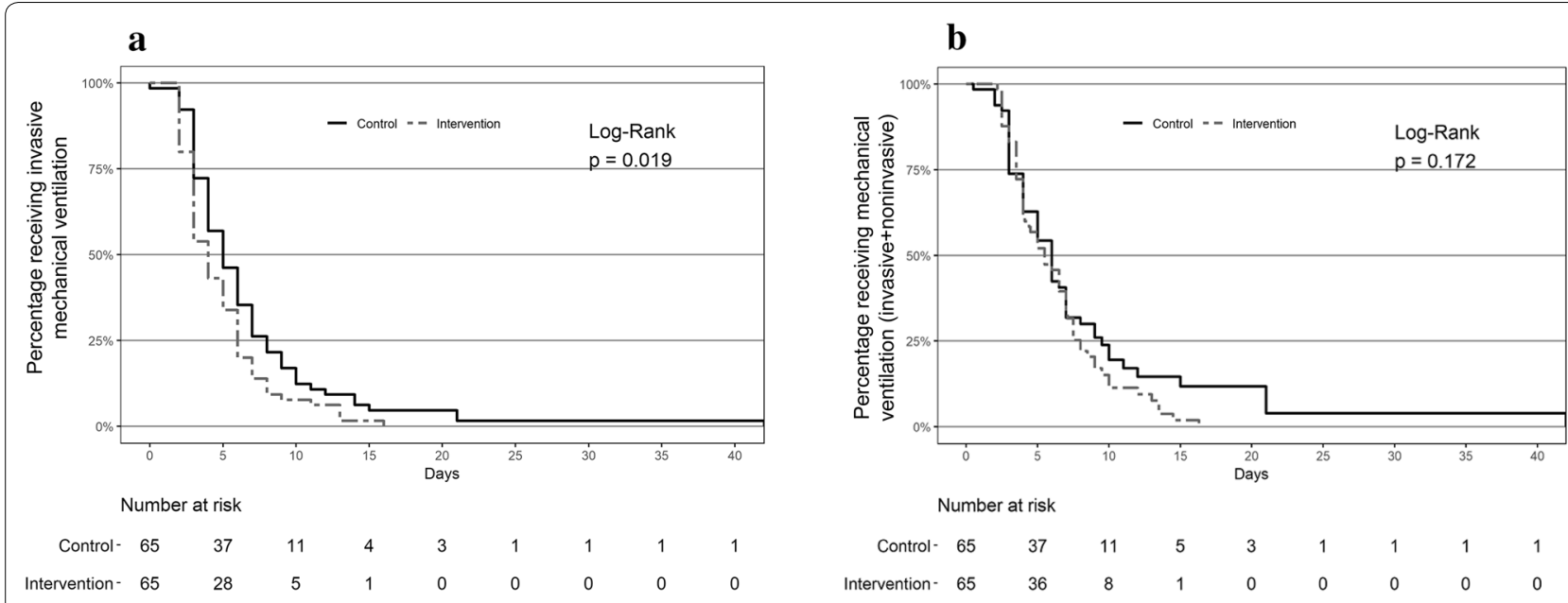

Fig. 3 Kaplan-Meier curves comparing time to liberation from invasive ventilation (a) and from any form of mechanical ventilation, invasive and noninvasive, (b) in control (solid line) and treatment (dashed line) groups

acute-on-chronic hypercapnic respiratory failure $[4,12$, 14-16]. A recent meta-analysis [7] showed that patients undergoing NIV after early extubation are characterized by fewer VAPs and tracheostomies, decreased duration of mechanical ventilation and intubation, ICU and hospital lengths of stay, and lower mortality. However, subgroup analysis indicated clear-cut benefits only for patients with acute-on-chronic respiratory failure, predominantly secondary to COPD exacerbation [7].

While strong evidence supports the use of NIV in patients with episodes of cardiogenic pulmonary edema [5], fewer and less robust data are available on NIV for other forms of hypoxemia in both immunosuppressed [17-19] and immunocompetent patients [20, 21]. In patients with hypoxemic ARF following abdominal surgery, NIV delivered via face mask has been shown to reduce the need for reintubation and for $\mathrm{i}-\mathrm{MV}$ and the number of episodes of health care-associated infection compared with standard oxygen therapy [6]. Although our patients were treated at a totally different ARF timing, a post hoc analysis showed that surgical patients benefited more than medical patients.

For patients recovering from hypoxemic ARF, only one single-center feasibility study has been published so far [8]. In 20 highly selected patients recovering from ARF, early extubation followed by immediate NIV application was feasible and safe. Despite these promising results, the paucity of patients makes any recommendation impossible [4]. In keeping with our previous pilot study, no patients received tracheostomy in the intervention group; however, only four patients (6\%) underwent tracheotomy, a much lower rate compared with $30 \%$ of patients in the control group of the pilot study. Compared with the aforementioned pilot study, the patients entering the present RCT also had lower median levels of PEEP at enrollment, 8 vs. $10.5 \mathrm{cmH}_{2} \mathrm{O}$, and shorter median ICU LOS, 8 vs.15.5 days. These differences might be a consequence of a selection bias or a consequence of having had only a single center in the previous pilot study, highly experienced in NIV, enrolling patients. Considering the accumulating evidence in favor of high-flow oxygen therapy (HFOT) through a nasal cannula for patients with hypoxemic ARF [22], some may argue that we should have considered HFOT rather than NIV. Nevertheless, at the time the study was designed, evidence on the role of HFOT [22-24] in hypoxemic patients was still missing. Moreover, since our patients were extubated early while dependent on both PEEP and PS, we still consider NIV the most proper strategy.

The interfaces utilized in this RCT partially differ from those adopted for the pilot study. In fact, while a standard helmet was used as first-line therapy in the pilot study, we allowed the use of a new helmet for this RCT. This interface has some benefits compared with the standard helmet as it more effectively delivers NIV with better comfort [25], pressurization [25-27], and triggering performance [25-27]. However, only $29 \%$ of the patients used this new helmet as first-choice interface. The probable reason for this limited use is the varying experience with this interface among centers. The rotational use of different interfaces is also a desirable practice that may improve patient comfort and limit side effects of the single interfaces [28]. In the pilot study, this strategy was adopted in $30 \%$ of the patients, while only 2 out of 65 patients $(3 \%)$ benefited from this strategy in the present 
study. The most likely explanation for this difference is related to the overall shorter time spent on NIV.

The reduction of $\mathrm{i}-\mathrm{MV}$ days and of some related side effects confirms the findings of studies where NIV was used to improve the process of withdrawing mechanical ventilation in hypercapnic patients [4, 7]. Nonetheless, we could not demonstrate a reduction in ICU LOS, suggesting that factors other than the duration of intubation, such as illness severity, reason of admission, age, comorbidities, and hospital stay before ICU admission, potentially influenced this outcome variable [29].

Some limitations of our study deserve discussion. First, the study includes a highly selected population of patients with hypoxemic ARF of varied etiology, as indicated by the low enrollment rate in relation to the relatively high numbers of recruiting centers, which indicates limited generalizability. Second, the rate of eligible patients that were effectively randomized was quite different between European and Chinese centers, i.e., 31\% vs. 7\%, respectively, which might lead to differences in the criteria for ICU admission between countries. In addition, the use of different interfaces, humidifiers, and ventilators may raise a concern about internal validity. Moreover, though we tried to minimize any bias that could influence internal validity at the study design stage, randomizing patients in blocks to prevent critical unbalancing and choosing centers with different NIV expertises, unfortunately our protocol made excluding unintended biases caused by the unblinded study design impossible, a limit that we were not able to overcome and that we share with all similar previous studies. Nevertheless, external validity should not be an issue considering that, differently from our previous small pilot investigation conducted in a highly experienced single center, the present study involves nine centers and includes many more patients. Finally, because various SBT techniques exist, we cannot exclude that treatment failure would have been different with diverse SBT techniques.

\section{Conclusions}

In highly selected patients recovering from an episode of hypoxemic non-hypercapnic ARF, the use of NIV to facilitate extubation, compared with standard treatment with the endotracheal tube in place, reduces the days spent on i-MV without affecting ICU length of stay. Furthermore, it is associated with fewer VAPs or VATs, fewer patients require sedation, and hospital LOS is shorter, with no differences in mortality. Although a post hoc analysis suggests that surgical patients are the best candidates for this approach, further studies are necessary to confirm these findings.
Electronic supplementary material

The online version of this article (https://doi.org/10.1007/s00134-018-5478-0) contains supplementary material, which is available to authorized users.

\begin{abstract}
Author details
1 Azienda Ospedaliero Universitaria "Maggiore Della Carità", Anestesia e Terapia Intensiva, Corso Mazzini 18, Novara, Italy. ${ }^{2}$ Università del Piemonte Orientale, via Solaroli 17, Novara, Italy. ${ }^{3}$ Ospedale Sant'Andrea, Anestesia e Rianimazione, Corso Abbiate 21, Vercelli, Italy. ${ }^{4}$ Emergency Department, Azienda Ospedaliera di Padova, Via Giustiniani 2, Padua, Italy. ${ }^{5}$ Department of Medicine, DIMED, University of Padova, Via Giustiniani 2, Padua, Italy. ${ }^{6}$ Department of Critical Care Medicine, Zhongda Hospital, School of Medicine, Southeast University, Nanjing 210009, Jiangsu, China. ${ }^{7}$ Department of Critical Care Medicine, The First Affiliated Hospital of Wannan Medical College, Wuhu 241001, Anhui, China. ${ }^{8}$ Department of Critical Care Medicine, The First Affiliated Hospital of Anhui Medical University, Hefei 230022, Anhui, China. ${ }^{9}$ Department of Critical Care Medicine, Xuzhou Central Hospital, Xuzhou 221009, Jiangsu, China. ${ }^{10}$ Anestesia e Rianimazione, Dipartimento di Scienze Mediche e Chirurgiche, Università "Magna Graecia", Viale Europa (Loc. Germaneto), Catanzaro, Italy. ${ }^{11}$ Department of Critical Care Medicine, Zhenjiang First People's Hospital, Zhenjiang 212002, Jiangsu, China. ${ }^{12}$ Department of Critical Care Medicine, Northern Jiangsu People's Hospital, Yangzhou 225000, Jiangsu, China. ${ }^{13}$ Azienda Sanitaria Locale del Verbano Cusio Ossola, Anestesia e Rianimazione, Piazza Vittime dei Lager Nazifascisti 1, Domodossola, Italy.

${ }^{14}$ Department of Biopathology and Medical Biotechnologies (DIBIMED), Section of Anesthesia, Analgesia, Intensive Care and Emergency, Policlinico Paolo Giaccone, University of Palermo, Via del Vespro 129, Palermo, Italy. ${ }^{15}$ Azienda Unità Sanitaria Locale di Reggio Emilia-IRCCS, S.C. Infrastruttura Ricerca e Statistica, Via Amendola 2, Reggio Emilia, Italy.
\end{abstract}

\section{Compliance with ethical standards}

\section{Conflicts of interest}

Dr. Gregoretti received fees for consulting or lectures from Philips, Merdtronic, Resmed, Vivisol, Orion Pharma, and Origin. Dr. Cavuto received fees from Intersurgical S.p.A. Dr. Navalesi's research laboratory has received equipment and grants from Maquet Critical Care, Draeger, and Intersurgical S.p.A. He also received honoraria/speaking fees from Maquet Critical Care, Orionpharma, Philips, Resmed, MSD, and Novartis. Dr Navalesi contributed to the development of the Next helmet, whose license for patent belongs to Intersurgical S.P.A., and receives royalties for that invention. Drs. Vaschetto, Longhini, Persona, Ori, Stefani, Liu, Yi, Lu, Yu, Luo, Tang, Li, Li, Cammarota, Bruni, Garofalo, Jin, Yan, Zheng, Yin, Guido, Della Corte, Fontana, Cortegiani, Giarratano, Montagnini, and Qiu declare that they do not have conflicts of interest.

\section{Ethical approval}

All procedures performed in the study were in accordance with the ethical standards of the institutional research committee and with the $1964 \mathrm{Hel}$ sinki Declaration and its later amendments or comparable ethical standards. Informed consent was obtained from all individual participants included in the study.

Received: 2 October 2018 Accepted: 20 November 2018 Published online: 10 December 2018

\section{References}

1. Esteban A, Frutos-Vivar F, Muriel A, Ferguson ND, Penuelas O, Abraira V, Raymondos K, Rios F, Nin N, Apezteguia C, Violi DA, Thille AW, Brochard L, Gonzalez M, Villagomez AJ, Hurtado J, Davies AR, Du B, Maggiore SM, Pelosi P, Soto L, Tomicic V, D’Empaire G, Matamis D, Abroug F, Moreno RP, Soares MA, Arabi Y, Sandi F, Jibaja M, Amin P, Koh Y, Kuiper MA, Bulow $\mathrm{HH}$, Zeggwagh AA, Anzueto A (2013) Evolution of mortality over time in patients receiving mechanical ventilation. Am J Respir Crit Care Med 188:220-230

2. Appendini L, Patessio A, Zanaboni S, Carone M, Gukov B, Donner CF, Rossi A (1994) Physiologic effects of positive end-expiratory pressure and mask 
pressure support during exacerbations of chronic obstructive pulmonary disease. Am J Respir Crit Care Med 149:1069-1076

3. L'her E, Deye N, Lellouche F, Taille S, Demoule A, Fraticelli A, Mancebo J, Brochard $L$ (2005) Physiologic effects of noninvasive ventilation during acute lung injury. Am J Respir Crit Care Med 172:1112-1118

4. Rochwerg B, Brochard L, Elliott MW, Hess D, Hill NS, Nava S, Navalesi PMOT, Antonelli M, Brozek J, Conti G, Ferrer M, Guntupalli K, Jaber S, Keenan S, Mancebo J, Mehta S, Raoof S Members Of The Task Force (2017) Official ERS/ATS clinical practice guidelines: noninvasive ventilation for acute respiratory failure. Eur Respir J 50:1602426. https://doi. org/10.1183/13993003.02426-2016

5. Masip J, Roque M, Sanchez B, Fernandez R, Subirana M, Exposito JA (2005) Noninvasive ventilation in acute cardiogenic pulmonary edema: systematic review and meta-analysis. JAMA 294:3124-3130

6. Jaber S, Lescot T, Futier E, Paugam-Burtz C, Seguin P, Ferrandiere M, Lasocki S, Mimoz O, Hengy B, Sannini A, Pottecher J, Abback PS, Riu B, Belafia F, Constantin JM, Masseret E, Beaussier M, Verzilli D, De JA, Chanques G, Brochard L, Molinari N (2016) Effect of noninvasive ventilation on tracheal reintubation among patients with hypoxemic respiratory failure following abdominal surgery: a randomized clinical trial. JAMA 315:1345-1353

7. Burns KE, Meade MO, Premji A, Adhikari NK (2014) Noninvasive ventilation as a weaning strategy for mechanical ventilation in adults with respiratory failure: a cochrane systematic review. CMAJ 186:E112-E122

8. Vaschetto R, Turucz E, Dellapiazza F, Guido S, Colombo D, Cammarota G, Della CF, Antonelli M, Navalesi P (2012) Noninvasive ventilation after early extubation in patients recovering from hypoxemic acute respiratory failure: a single-centre feasibility study. Intensive Care Med 38:1599-1606

9. Altman DG, Schulz KF, Moher D, Egger M, Davidoff F, Elbourne D, Gotzsche PC, Lang T (2001) The revised CONSORT statement for reporting randomized trials: explanation and elaboration. Ann Intern Med 134:663-694

10. Dellinger RP, Levy MM, Rhodes A, Annane D, Gerlach H, Opal SM, Sevransky JE, Sprung CL, Douglas IS, Jaeschke R, Osborn TM, Nunnally ME, Townsend SR, Reinhart K, Kleinpell RM, Angus DC, Deutschman CS, Machado FR, Rubenfeld GD, Webb SA, Beale RJ, Vincent JL, Moreno R (2013) Surviving sepsis campaign: international guidelines for management of severe sepsis and septic shock: 2012. Crit Care Med 41:580-637

11. Nava S, Gregoretti C, Fanfulla F, Squadrone E, Grassi M, Carlucci A, Beltrame F, Navalesi P (2005) Noninvasive ventilation to prevent respiratory failure after extubation in high-risk patients. Crit Care Med 33:2465-2470

12. Ferrer M, Esquinas A, Arancibia F, Bauer TT, Gonzalez G, Carrillo A, Rodriguez-Roisin R, Torres A (2003) Noninvasive ventilation during persistent weaning failure: a randomized controlled trial. Am J Respir Crit Care Med 168:70-76

13. Khwaja A (2012) KDIGO clinical practice guidelines for acute kidney injury. Nephron Clin Pract 120:c179-c184

14. Girault C, Daudenthun I, Chevron V, Tamion F, Leroy J, Bonmarchand G (1999) Noninvasive ventilation as a systematic extubation and weaning technique in acute-on-chronic respiratory failure: a prospective, randomized controlled study. Am J Respir Crit Care Med 160:86-92

15. Girault C, Bubenheim M, Abroug F, Diehl JL, Elatrous S, Beuret P, Richecoeur J, L'her E, Hilbert G, Capellier G, Rabbat A, Besbes M, Guerin C, Guiot P, Benichou J, Bonmarchand G (2011) Noninvasive ventilation and weaning in patients with chronic hypercapnic respiratory failure: a randomized multicenter trial. Am J Respir Crit Care Med 184:672-679

16. Nava S, Ambrosino N, Clini E, Prato M, Orlando G, Vitacca M, Brigada P, Fracchia C, Rubini F (1998) Noninvasive mechanical ventilation in the weaning of patients with respiratory failure due to chronic obstructive pulmonary disease. A randomized, controlled trial. Ann Intern Med 128:721-728

17. Antonelli M, Conti G, Bufi M, Costa MG, Lappa A, Rocco M, Gasparetto A, Meduri GU (2000) Noninvasive ventilation for treatment of acute respiratory failure in patients undergoing solid organ transplantation: a randomized trial. JAMA 283:235-241

18. Hilbert G, Gruson D, Vargas F, Valentino R, Gbikpi-Benissan G, Dupon M Reiffers J, Cardinaud JP (2001) Noninvasive ventilation in immunosuppressed patients with pulmonary infiltrates, fever, and acute respiratory failure. N Engl J Med 344:481-487

19. Lemiale V, Mokart D, Resche-Rigon M, Pene F, Mayaux J, Faucher E, Nyunga M, Girault C, Perez P, Guitton C, Ekpe K, Kouatchet A, Theodose I, Benoit D, Canet E, Barbier F, Rabbat A, Bruneel F, Vincent F, Klouche K, Loay K, Mariotte E, Bouadma L, Moreau AS, Seguin A, Meert AP, Reignier J, Papazian L, Mehzari I, Cohen Y, Schenck M, Hamidfar R, Darmon M, Demoule A, Chevret S, Azoulay E (2015) Effect of noninvasive ventilation vs oxygen therapy on mortality among immunocompromised patients with acute respiratory failure: a randomized clinical trial. JAMA 314:1711-1719

20. Delclaux C, L'Her E, Alberti C, Mancebo J, Abroug F, Conti G, Guerin C, Schortgen F, Lefort Y, Antonelli M, Lepage E, Lemaire F, Brochard L (2000) Treatment of acute hypoxemic nonhypercapnic respiratory insufficiency with continuous positive airway pressure delivered by a face mask: a randomized controlled trial. JAMA 284:2352-2360

21. Ferrer M, Esquinas A, Leon M, Gonzalez G, Alarcon A, Torres A (2003) Noninvasive ventilation in severe hypoxemic respiratory failure: a randomized clinical trial. Am J Respir Crit Care Med 168:1438-1444

22. Renda T, Corrado A, Iskandar G, Pelaia G, Abdalla K, Navalesi P (2018) High-flow nasal oxygen therapy in intensive care and anaesthesia. $\mathrm{Br} J$ Anaesth 120:18-27

23. Frat JP, Thille AW, Mercat A, Girault C, Ragot S, Perbet S, Prat G, Boulain T, Morawiec E, Cottereau A, Devaquet J, Nseir S, Razazi K, Mira JP, Argaud L, Chakarian JC, Ricard JD, Wittebole X, Chevalier S, Herbland A, Fartoukh M, Constantin JM, Tonnelier JM, Pierrot M, Mathonnet A, Beduneau G, Deletage-Metreau C, Richard JC, Brochard L, Robert R (2015) High-flow oxygen through nasal cannula in acute hypoxemic respiratory failure. $\mathrm{N}$ Engl J Med 372:2185-2196

24. Maggiore SM, Idone FA, Vaschetto R, Festa R, Cataldo A, Antonicelli F, Montini L, De GA, Navalesi P, Antonelli M (2014) Nasal high-flow versus venturi mask oxygen therapy after extubation. Effects on oxygenation, comfort, and clinical outcome. Am J Respir Crit Care Med 190:282-288

25. Olivieri C, Longhini F, Cena T, Cammarota G, Vaschetto R, Messina A Berni P, Magnani C, Della CF, Navalesi P (2016) New versus conventional helmet for delivering noninvasive ventilation: a physiologic, crossover randomized study in critically ill patients. Anesthesiology 124:101-108

26. Olivieri C, Costa R, Spinazzola G, Ferrone G, Longhini F, Cammarota G, Conti G, Navalesi P (2013) Bench comparative evaluation of a new generation and standard helmet for delivering non-invasive ventilation. Intensive Care Med 39:734-738

27. Vaschetto R, De Jong A, Conseil M, Galia F, Mahul M, Coisel Y, Prades A, Navalesi P, Jaber S (2014) Comparative evaluation of three interfaces for non-invasive ventilation: a randomized cross-over design physiologic study on healthy volunteers. Crit Care 18:R2-

28. Nava S, Navalesi P, Carlucci A (2009) Non-invasive ventilation. Minerva Anestesiol 75:31-36

29. Verburg IWM, Holman R, Dongelmans D, de Jonge E, de Keizer NF (2018) Is patient length of stay associated with intensive care unit characteristics? J Crit Care 43:114-121 